\title{
Variable structure Control Applied to Water Supply
}

\author{
Dr. Volodymyr Sydorenko ${ }^{1}$, Kostyantyn Buravchenko ${ }^{2}$ \\ ${ }^{1,2}$ (Department of Programming and Information Protection, Kirovohrad National Technical University, \\ Ukraine)
}

\begin{abstract}
This paper describes the controller with variable structure in water supply management system. It is shown that in the transition mode it should be used the bang-bang controller. In stabilization mode problem is solved using linear-quadratic regulator. In the article block diagram of control system was proposed, which implements an optimal strategy in two different modes for the water system.
\end{abstract}

Keywords: variable structure control, water supply, linear quadratic regulator, control system

\section{Introduction}

Water supply is distributed stochastic system in which oscillatory processes occur due to uneven water consumption [1], and which significantly affect by pipeline options [2]. In modern control systems there are using frequency converters that automatically maintain a preset pressure in the pipeline. Changing modes of water systems require complex control system pressure in order to ensure optimal energy consumption at a given performance and established a transitional process. One of the promising areas of research is the theory of sliding control. Why sliding system - because they make it possible to apply the benefits of optimal performance and optimal linear quadratic regulators in one management system. In the article was proposed to investigate the possibility of using variable structure regulator applied to water supply control system.

2.1 Motivation and Background

\section{Structured Optimal Control}

Let us consider the water supply system, which describes by the system of differential equation:

$$
\left\{\begin{array}{l}
\dot{x}(t)=A(t) x(t)+B(t) u(t), \\
y(t)=C x(t)
\end{array}\right.
$$

with $\mathrm{A}(t)$ is the matrix, $\mathrm{n} \times \mathrm{n} ; \mathrm{B}(t)$ is the matrix $\mathrm{n} \times \mathrm{m} ; x(t)$ is the vector output $\mathrm{n} \times 1$; the $\mathrm{u}(t)$ - vector of control signal $\mathrm{m} \times 1, y(t)$ - is the vector of the measuring coordinates of water system $(m \times 1) ; C(t)$ - matrix $(m \times n)$;

The task of the management system is to change the control signal $\mathrm{u}(t)$ to minimize error $\varepsilon(t)$, the difference between the measured $\mathrm{y}(t)$ signal and the necessary signal $\hat{y}(t)$ :

$$
\varepsilon(t)=\hat{y}(t)-y(t) \rightarrow \min
$$

As described in $[2,3,4]$, it's need to find a control signal that minimizes the cost function

$$
J(\mathbf{x}, \mathbf{u})=\frac{1}{2} \int_{t_{0}}^{t_{k}}\left[\mathbf{x}^{T}(t) \mathbf{Q}(t) \mathbf{x}(t)+\mathbf{u}^{T}(t) \mathbf{R}(t) \mathbf{u}(t)\right] d t+\frac{1}{2} \mathbf{x}^{T}\left(t_{k}\right) \mathbf{S} \mathbf{x}\left(t_{k}\right),
$$

with $\mathbf{Q}, \mathbf{R}, \mathbf{S}$ are symetric matricies; $\mathbf{R}$ is the positively defined matrix; $\mathbf{Q}, \mathbf{S}$ is the positively semidefinite matrix; $J(\varepsilon, u)$ - cost function, $L_{\varepsilon}=\frac{1}{2} \int_{t_{0}}^{t_{k}}\left[\varepsilon^{T}(t) Q \varepsilon(t)\right] \mathrm{d} t$ - the penalty for deviation for the whole period of control, $L_{u}=\frac{1}{2} u^{T}(t) R u(t)$ - the penalty of energetic usage at control process, $L_{s}=\frac{1}{2} \varepsilon^{T}\left(t_{k}\right) S \varepsilon\left(t_{k}\right)$ - the penalty for deviation at the end of control.

Recommendations on selection matrix $Q$ and $R$ :

$$
\begin{array}{r}
q_{i i}=\left(\frac{x_{1 \max }}{x_{i \max }}\right)^{2} q_{11} ; r_{j j}=\left(\frac{u_{1 \max }}{u_{i \max }}\right)^{2} r_{11}, \\
\sum_{i=1}^{n} q_{i i} x_{i \max }^{2}=\sum_{j=1}^{k} r_{j j} u_{j \max }^{2} .
\end{array}
$$

In the water supply system the work mode often changes(Fig. 1), due to stochastic and uneven water consumption, which is considered in detail in [1]. 


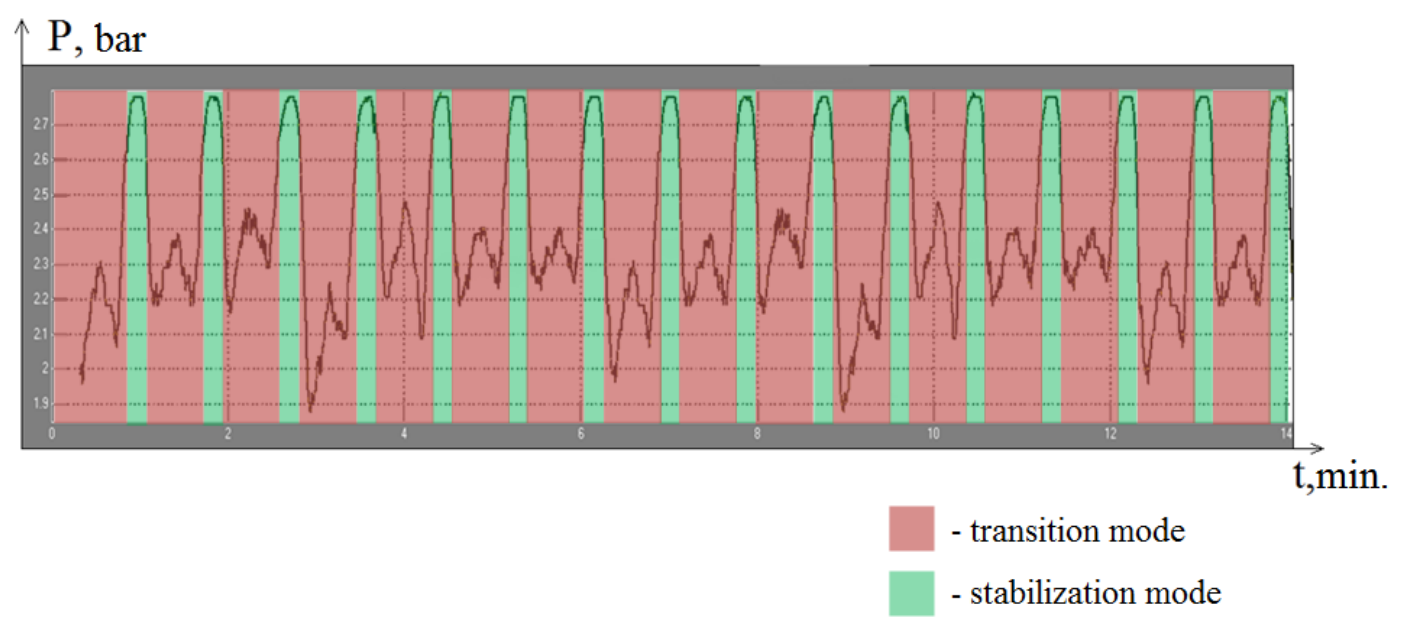

Fig. 1 - Work modes changing in time in water supply system

Depending on the work mode of the water supply system, the problem of control system is: how we need to change the regulator. Let describe two different modes.

\subsection{Transition Mode}

The task of the control system in a transient mode is to transfer vector of system state $\mathrm{y}(t)$ from point $P_{0}$ to point $P_{1}$ in minimum time (time-optimal control $\left.[5,6,7]\right) . P_{0}$ - this is the point at which the water system came as a result of hopping pressure change. $P_{1}$ - setpoint state of the water supply system, which must be maintained. Based on the theory of minimum time problem in the transition process it should be used driving with discrete functions [5]. Otherwise equation (1) can be represented as a homogeneous system of first order differential equations.

$$
\left\{\begin{array}{c}
\dot{x}_{1}=f_{1}\left(x_{1}, \ldots, x_{n}, u_{1}, \ldots, u_{n}, t\right) \\
\ldots \ldots \ldots . . . \\
\dot{x}_{n}=f_{n}\left(x_{1}, \ldots, x_{n}, u_{1}, \ldots, u_{n}, t\right)
\end{array}, x\left(t_{0}\right)=x_{0}, t_{0} \leq t \leq t_{k},\right.
$$

According to the maximum principle, we need to define a costate system of differential equations, which in vector form presented by:

$$
\dot{\Psi}=-A^{T} \Psi(t)
$$

where $\Psi(t)$ - costate vector $(n \times 1)$;

Relation between systems (6) and (7) sets Hamiltonian:

$$
H\left(X, U, t, \Psi, \Psi_{0}\right)=\Psi_{0} f_{0}(X, U, t)+\Psi_{1} f_{1}(X, U, t)+\ldots+\Psi_{n} f_{n}(X, U, t)=\sum_{j=1}^{n} \Psi_{i} f_{i}(X, U, t)
$$

For the optimal system dynamics it is needed to follow Pontryagin maximum principle.

$$
\begin{gathered}
\max H\left(X, U, t, \Psi, \Psi_{0}\right)=H\left(x(t), u^{*}(t), t, \Psi, \Psi_{0}\right), \\
u^{*}=U\left(t, X, \Psi, \Psi_{0}\right)
\end{gathered}
$$

Construction of regulator for the transition mode of water supply considered in [6].

\subsection{Steady-state Mode (Stabilization Mode)}

The problem of constructing a regulator in stabilization mode was discussed in detail in [8]. It was shown that the optimal control $u^{*}(t)$ in stabilization mode with minimal energy consumption determined by the equation of the form

$$
u^{*}(t)=-R^{-1}(t) B^{T} P(t) x(t),
$$

where $P(t)$ must satisfy the solve of matrix differential equation Riccati type. It causes problems when for calculation we use on-board microcontrollers.

To minimize functional (3) the $K(t)$ defines as follows:

$$
K(t)=-R^{-1}(t) B^{T} P(t)
$$


equation $u^{*}(t)$ transfer as follows

$$
u^{*}(t)=K(t) x(t)
$$

\section{Variable Structure Regulator}

Based on the proposed ideas it is necessary to create a management system that would include two regulator and switching unit, which depends on the selection of the required controller in two modes. Functional diagram of the controller shown in Figure 2.

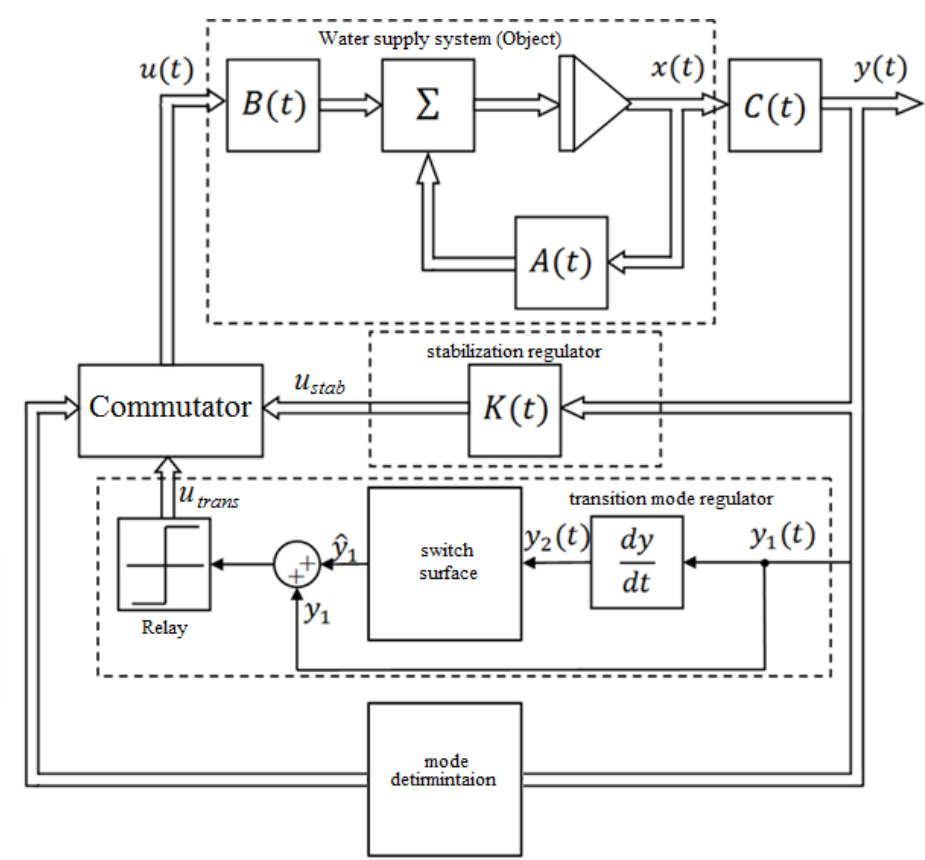

Fig. 2 - Functional diagram of the controller with variable structure

The system includes the advantages of two types of optimal controllers (the optimal time and linear quadratic), which is an important variable in terms of water consumption, and as a consequence of changes in operating conditions.

\subsection{An example}

For example, consider pumping unit that has the following options:

$T_{e m}=0.026$ - electromechanical time constant of asynchronous motor with squirrel cage;

$T_{n}=0.231-$ time constant pump;

$k_{e m}=5.061$ - engine gain;

$k_{n}=5.22-$ pump gain.

$T_{1}=T_{e m} T_{n}=0.006006, T_{2}=T_{e m}+T_{n}=0.257, k=k_{e m} k_{n}=26.41842$.

If you use only PID controller, the control scheme of variable structure takes the following form (Fig. 2).

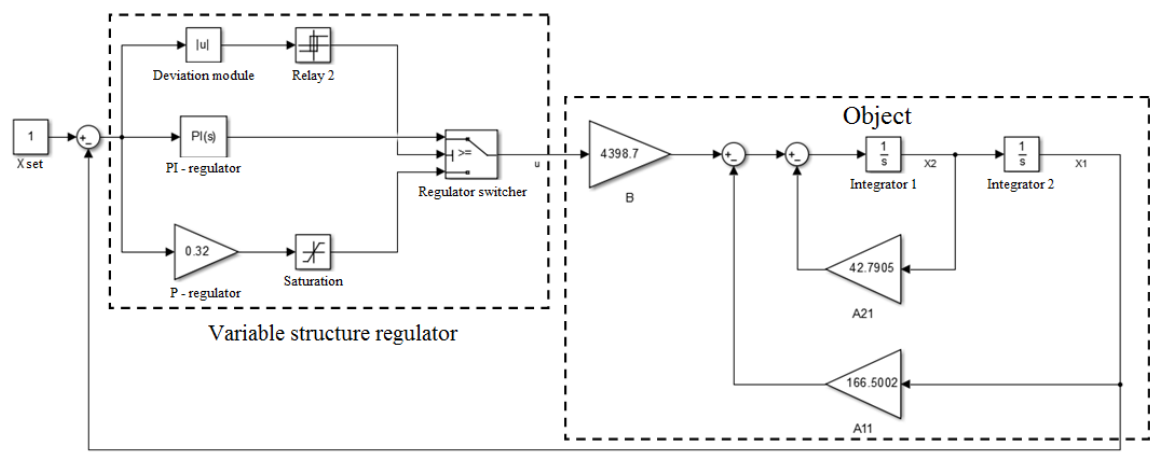

Fig. 2 - Variable structure regulator with $\mathrm{P}$ and PI controller. 
In this case, the P-controller was chosen in the transition mode, in steady-state mode we were using PIcontroller. P-controller provides fast transition process, but the drawback is that there are oscillations . PI controller stabilizes only pressure. These controllers are one-dimensional and do not considers whole status of the system. Control process diagram shown in Figure 3.

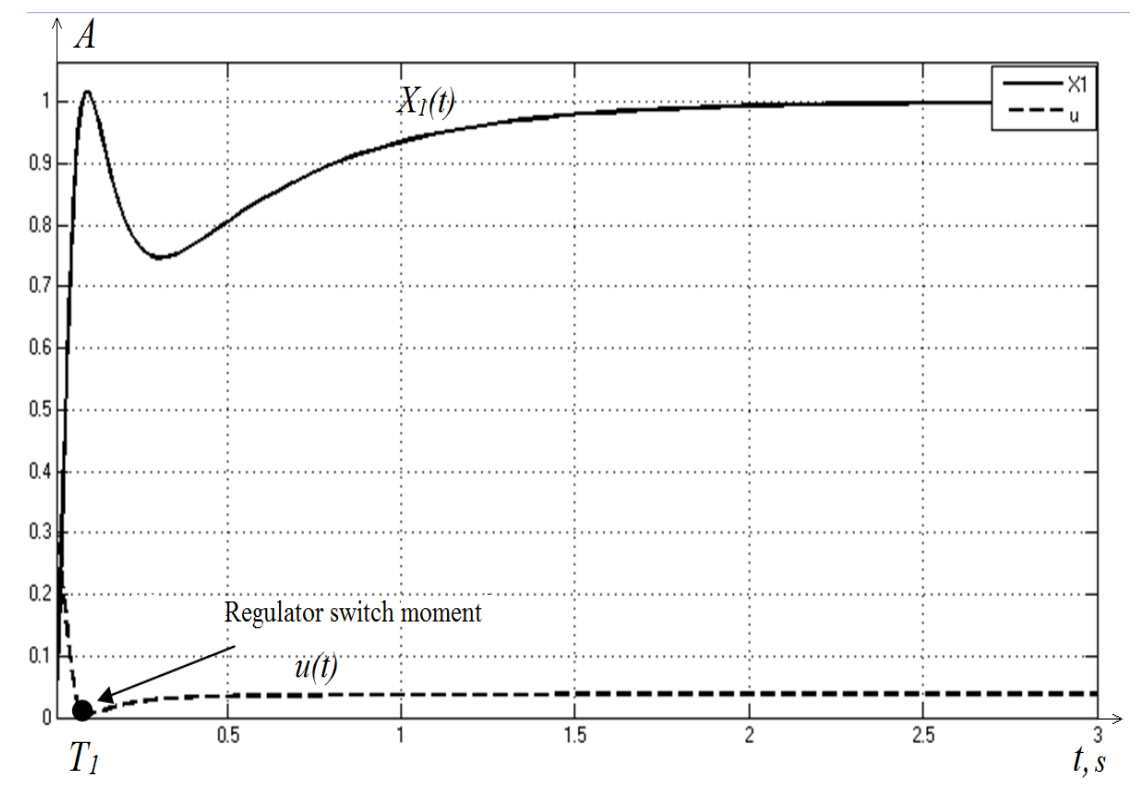

Fig. 3 - Control process diagram

This diagram is built, while the PI and P coefficient corresponds to linear-quadratic functional minimum (J).

The optimal controller with variable structure shown in Figure 4.

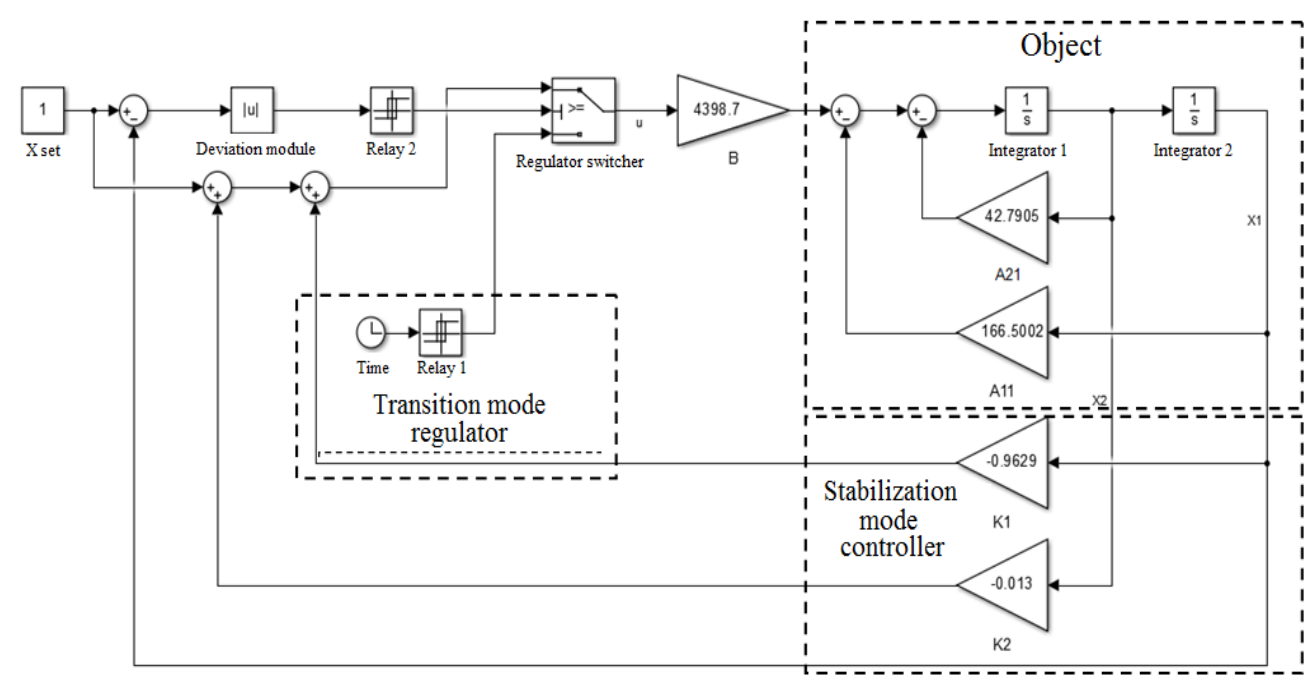

Fig. 4 - Variable structure regulator with optimal controller.

Gain $\mathrm{K}_{1}$ and $\mathrm{K}_{2}$ of the LQR determined using [8].

The principle of the control system is as follows: relay 2 receives input deviation from the set pressure. If the deviation exceeds a given threshold, then the controller switches to the stabilization regulator after transition mode. Regulator of transition mode provides minimal recovery time to stabilization mode.

Diagram system is shown in Figure 


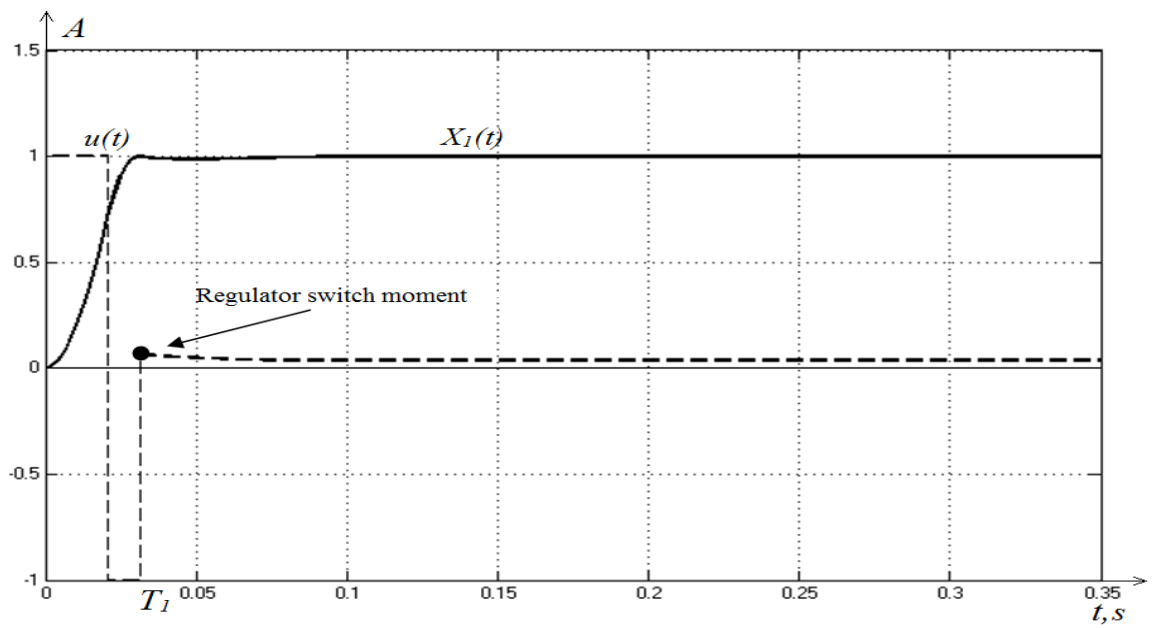

\section{Problems}

The problem of determining the moment of switching control action $\mathrm{u}(t)$ in the transition mode remains unsolved. It is known, that to solve this problem, it is need to define the initial conditions $\Psi_{0}$ for the function $\psi(t)$. For this system should be at the entrance to the transition mode to perform delay and find $P_{0}$ point from which to start for optimum speed control.

Besides the previous problem definition points actually we need to find moment to switch regulators. Switching unit based on state property shall determine the mode in which it came. For real objects such as water supply system, a condition which can be called transition determined from the physical limitations for a system, such as the efficiency of the pump, operating point system in which consumers get a given volume of water with a given pressure. For each system there are regulations that describe all the necessary requirements to work. In practice, the implementation of the rules will define modes, though for different systems requirements are clearly different.

The disadvantage of the system under consideration is that it ignores the disturbance consumption and pressure fluctuations, which planned to introduce a mathematical model for further studies.

\section{Conclusion}

To solve the problem of water supply management system in various modes it is proposed to use the controller with variable structure. Changing regulators in the corresponding modes can more accurately perform the task that is placed in this mode. In transition mode control system uses optimal-time regulator and in stabilization mode - linear-quadratic regulator. There is a problem determining regulators switching points. It is noted that the detection time of switching is entirely dependent on the physical characteristics of an object and requires further research.

\section{References}

[1]. Sydorenko, V.V, Buravchenko K.O., Analiz prychyn kolyvannia tysku u systemakh vodopostachannia z metoiu yikh minimizatsii [Analysis of the Causes of Pressure Fluctuations in Water Supply Systems]. Zbirnyk naukovykh prats. Natsionalnyi universytet korablebuduvannia imeni admirala Makarova, 28 (460), 2015, pp. 113-117.

[2]. Buravchenko, K. O. Research and analysis of dynamics of pump unit control process. Technology audit and production reserves, 3(2 (29)), 2016, 15-19.

[3]. Bakker, M., Rajewicz, T., Kien, H., Vreeburg, J. H. G., and Rietveld, L. C., Reducing energy consumption and leakage by active pressure control in a water supply system. In ICEAM 2013: International Conference of Economics and Asset Management" Asset Management for Enhancing Energy Efficiency in Water and Wastewater Systems", Marbella, Spain, 24-26 April 2013. IWA.

[4]. Araujo, L. S., Ramos, H., and Coelho, S. T., Pressure control for leakage minimisation in water distribution systems management. Water Resources Management, 20(1), 2006, 133-149.

[5]. Pontryagin, L. C., Boltyansky, V. C., Gamkrelidze, R. V., and Mischenko, E. F. (1961). Mathematical theory of optimal problem. Gosudarst. Izdat. Fiz.-Mat. Literatury, Moskva, Nauka (in Russian).

[6]. Sydorenko, V.V, Buravchenko K.O.. Systema keruvannya vodopostachannyam z artezians'kykh sverdlovyn u perekhidnomu rezhymi [The control system of water supply from boreholes in the transition mode]. ScienceRise, 6(2 (23)), 2016, 45-50.

[7]. Jung, D., Kang, D., Kang, M., and Kim, B.,Real-time pump scheduling for water transmission systems: Case study. KSCE Journal of Civil Engineering,19(7), 2015, 1987-1993.

[8]. Sidorenko, V. V., and Buravchenko, K. O. Optimal Control in Water Supply Condition Stabilization Mode. Electronics and control systems, (1), 2016, 50-53. 